V. YANOVSKY

C. FELIX

G. MOUROU

\section{Why ring regenerative amplification (regen)?}

Center for Ultrafast Optical Science, University of Michigan, 2200 Bonisteel Blvd., Ann Arbor, MI 48109-2099, USA

\section{Received: 17 September 2001/}

Revised version: 24 October 2001

Published online: 5 July 2002 • (C) Springer-Verlag 2002

ABSTRACT We show that ring cavity regenerative amplifiers (regens) have distinct advantages over the linear ones for applications in chirped pulse amplification. Larger energy, better contrast and better isolation from the oscillator are experimentally demonstrated.

PACS 42.65.Re

\section{1}

\section{Introduction}

In modern high-power chirped-pulse amplification (CPA) Ti:sapphire lasers, a femtosecond pulse of a master oscillator is stretched to subnanosecond pulsewidth and is subsequently amplified to joule scale energy by using either several stages of multipass amplifiers or a combination of a regenerative amplifier (regen) and multipass amplifiers. Stable linear cavities are mostly utilized in regenerative amplifiers although an unstable one was also reported [1]. Ring cavities, being more complicated and harder to align comparing to linear ones, do not have widespread use, however they have several inherent advantages over linear designs for applications in CPA lasers. The important aspects to consider when choosing a design are (among others) the output energy, contrast and isolation from the oscillator. Ring cavities are preferable in these respects, as we demonstrate below.

2

\section{Energy}

A typical meter-size linear Ti:sapphire regenerative amplifier has submillimeter mode size and output energy of $1-10 \mathrm{~mJ}$ limited by the saturation fluence of Ti:sapphire $\left(0.9 \mathrm{~J} / \mathrm{cm}^{2}\right)$ and intracavity losses. Subsequent amplification to joule scale energy requires a saturated gain of 100-1000 which is hard to reach with a single multipass amplifier, so multiple stages of multipass amplifiers are used resulting in beam quality degradation and overall complexity of the laser system. It is therefore important to maximize the regenerative amplifier output energy. Increasing the regenerative amplifier

Fax:+1-734/763-4876, E-mail: vpy@eecs.umich.edu output energy implies increasing a mode size. For a stable resonator larger mode size leads to larger cavity length. Using a ring design for a long resonator is advantageous, as a ring cavity (with the same mode size as a linear one) can have a smaller length. Although mode size does not directly depend on cavity geometry (ring or linear), a choice of a ring cavity allows to use resonator designs which cannot be used in linear configuration. One example is a hemispherical linear resonator. It has the same mode size and half the length of a spherical resonator with mirrors of the same curvature but is unpractical for high-energy applications because it has a small beam waist on a plain mirror. In a spherical ring cavity (spherical and hemispherical ring cavities are identical) the plain mirror is replaced with several mirrors which could be placed away from the beam waist. The length of such a cavity is half the length of the linear spherical cavity with the same curved mirrors. As an example, consider a ring all-reflective-optics regenerative amplifier [2] we developed for the high-power (> $100 \mathrm{TW}$ ) Ti:sapphire laser we are currently building.

The cavity (Fig. 1) is close to being spherical, however the resonator roundtrip length $(7.5 \mathrm{~m})$ is less than twice the radius of curvature of the curved mirror $(4 \mathrm{~m})$ providing for cavity stability. The Ti:sapphire crystal $(2 \mathrm{~cm}$ diameter, $1 \mathrm{~cm}$ length, $0.15 \%$ doping) is located close to the curved mirror where the beam size is the largest. This arrangement allows the amplified beam to double-pass the gain region, eliminating the common disadvantage of a ring cavity (one gain-length per roundtrip). The beam waist is far from optical elements to insure a high damage threshold. A double peak Pockels cell (Thomson-CSF) injects the pulse in the cavity and ejects it after gain saturation is reached. Broadband $(200 \mathrm{~nm})$ thin film polarizers and high reflectors ensure that the output bandwidth is limited only by the gain narrowing. Roundtrip cavity losses are $16 \%$. By pumping the regen (regenerative amplifier) with 80-340 mJ absorbed energy of the frequencydoubled Nd:YAG pump laser, we are able to reach an output energy of up to $90 \mathrm{~mJ}$ at $37 \%$ slope efficiency (Fig. 2). Al-

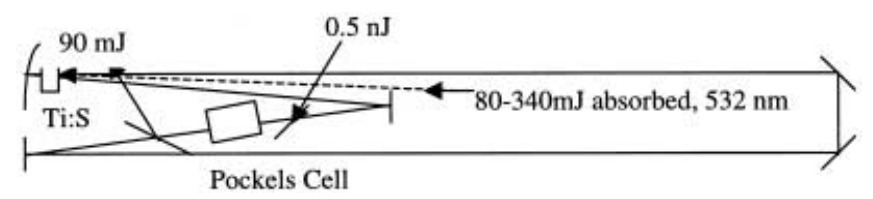

FIGURE 1 Regenerative amplifier layout 


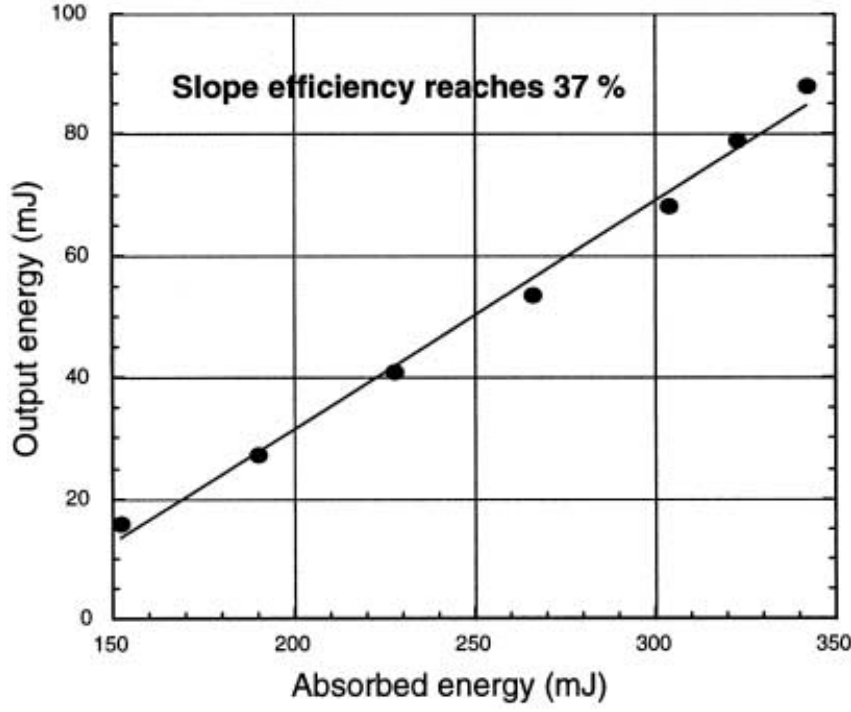

FIGURE 2 Regenerative amplifier output energy vs. pump energy

lowing for $80 \%$ output polarizer efficiency for the rejected polarization, the intracavity energy is $110 \mathrm{~mJ}$. This value of the intracavity energy is limited by optical breakdown of air (caused by dust particles) in the beam waist at higher energies. The injected stretched pulse width is $\sim 1 \mathrm{~ns}$, the output bandwidth is $45 \mathrm{~nm}$ (FWHM). The slope efficiency of the amplifier is 0.37 (Fig. 2).

This value matches well the slope efficiency values $\eta=0.39-0.38$ estimated using the following formula [2]

$\eta=\eta_{\mathrm{e}} \lambda_{\mathrm{p}} / \lambda \exp \left(-\tau_{\mathrm{p}} n / \tau\right) R_{\mathrm{p}}$

where $\lambda_{\mathrm{p}} / \lambda=0.66$ is the ratio of the pump wavelength to the lasing wavelength, $R_{\mathrm{p}}=0.8$ the output polarizer reflectivity, $\tau_{\mathrm{p}}=25 \mathrm{~ns}$ the cavity roundtrip, $\tau=3.2 \mu$ s the Ti:sapphire lifetime, $n \sim 20-30$ the number of roundtrips necessary to reach the peak fluence, $\eta_{\mathrm{e}}$ the slope extraction efficiency defined as the derivative of the peak output fluence $J_{\mathrm{p}}$ by stored energy per unit area $J_{\mathrm{A}}=2 J_{\mathrm{s}} \ln G_{0}$ :

$\eta_{\mathrm{e}}=\partial J_{\mathrm{p}} / \partial J_{\mathrm{A}}=1-G_{0}(1-T) /\left(G_{0}-1\right) \quad$,

where $J_{\mathrm{s}}$ is the saturation fluence, $T$ the roundtrip transmission, $G_{0}$ the unsaturated roundtrip gain and $J_{0}$ the input fluence.

In spite of the large roundtrip length the cavity has a modest footprint of $1 \mathrm{~m}^{2}$ and exhibits exceptionally good alignment stability. We were able to run the regen for months without need for mirror adjustment. This is due partly to a lower sensitivity of a ring cavity to misalignment compared with a linear cavity.

3

\section{Contrast}

Leakage through a switchout polarizer prior to the switchout pulse and amplified spontaneous emission (ASE) limit the intensity contrast of a regenerative amplifier. While the leakage can be removed by an external Pockels cell, the ASE contribution is almost impossible to remove, because a part of ASE temporally overlaps with a stretched pulse. In

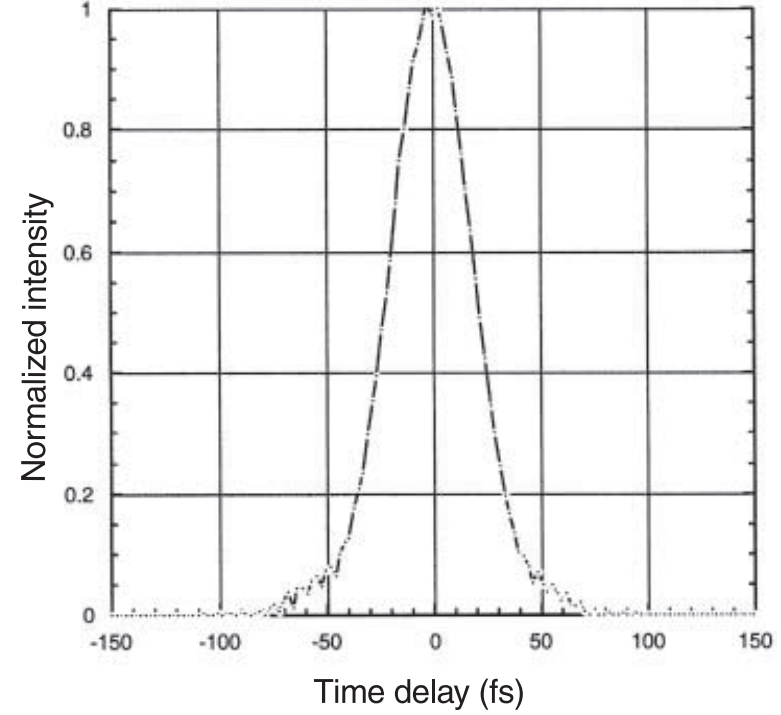

FIGURE 3 Autocorrelation of the 32 fs FWHM (assuming Gaussian pulse shape) output pulse of the regenerative amplifier

the approximation $G_{0}>1$ ASE flux, $I_{\mathrm{ASE}}$ can be calculated as [3]

$I_{\mathrm{ASE}}=J_{\mathrm{S}} / \tau \Omega / 4 G_{0} /\left(\ln G_{0}\right)^{1 / 2}$,

where $\Omega$ is the ASE solid angle. For a resonator mode $\Omega \sim$ $\omega^{2} / L^{2}$, where $\omega$ is a mode size and $L$ the cavity length. On the other hand, for a Gaussian mode $\omega^{2} \sim L \lambda \mathrm{g}$ resulting in $\Omega \sim \lambda / L$. It follows from (3) that $I_{\mathrm{ASE}} \sim 1 / L$, meaning that ring cavities have higher intensity contrast relative to ASE compared to linear ones with the same footprint. In addition, only ASE going into the direction of the main pulse matters in a ring cavity while both directions could contribute in a linear cavity, adding another factor of 2 . For example, the cavity described above should have an ASE intensity contrast a factor of 15 larger compared to a meter-long linear cavity. We measured the energy contrast of the ring regenerative amplifier described above using a fast photodiode. The ASE pulse was about $2 \mathrm{~ns}$ long and had $\sim 0.1 \%$ of the main pulse energy, leading to an intensity contrast of $\sim 10^{8}$ between ASE and the $\sim 30$-fs-long main pulse (after compression, Fig. 3). For comparison, reported values of ASE contrast range from $10^{6}$ [4] for a linear regen to $10^{7}$ for a multipass amplifier [5].

\section{$4 \quad$ Isolation from oscillator}

Kerr-lens mode-locked oscillators are rather sensitive to a backreflected output beam and stop mode-locking at excessive backreflection. Due to the nature of the injection, the backreflected beam cannot be misaligned to miss the oscillator input aperture. Typically, no more than picojoule levels of the backrefleted energy can be tolerated. A ring and a linear cavity differ dramatically in the amount of energy they send back to the oscillator. With a typical linear regen energy of at least $1 \mathrm{~mJ}$ and a switch-in polarizer isolation of $\sim 1 \%$, approximately $10 \mu \mathrm{J}$ is expected to go back into the oscillator. In order to suppress this level of backreflection two stages of isolation (Pockels cells or Faraday rotators) are needed, because 
one isolation stage cannot provide isolation of 6-7 orders of magnitude. In contrast to the case of a linear cavity, a ring cavity sends back to the oscillator only ASE and one stage of isolation is sufficient. In the example discussed above, not only did we just use a single Pockels cell to isolate the regen from the oscillator, but we also have an additional two-pass amplifier with a small signal gain on the order of 100 between the regen and the oscillator without any detrimental effects on the oscillator performance.

ACKNOWLEDGEMENTS This work was supported by the National Science Foundation.

\section{REFERENCES}

1 C. LeBlanc, P. Curley, F. Salin: "High energy regenerative amplification of femtosecond pulses using gain guiding". In: OSA Proc. Advanced Solid-State Lasers, Vol. 24 (Optical Society of America, Washington, DC 1995) pp. 314-316

2 V. Yanovsky, C. Felix, G. Mourou: IEEE J. Select. Topics Quant. Electr. 7, $539(2001)$

3 W. Koechner: Solid State Laser Engineering (Springer-Verlag, Berlin, Heidelberg, New York 1996)

4 K. Yamakawa, C. Barty: IEEE J. Select. Topics Quant. Electr. 6, 658 (2000)

5 J.P. Chambaret, C. Le Blanc, G. Cheriaux, P. Curley, G. Darpentigny, P. Rousseau, G. Hamoniaux, A. Antonetti, F. Salin: Opt. Lett. 21, 1921 (1996) 